\title{
EL TERREMOTO DE SÁMARA (COSTA RICA) DEL 5 DE SETIEMBRE DEL 2012 (Mw7,6)
}

\author{
THE SAMARA (COSTA RICA) EARTHQUAKE OF 5 SEPTEMBER 2012 (Mw 7.6)
}

\section{Lepolt Linkimer ${ }^{1 *}$, Ivonne Arroyo ${ }^{2}$, Mauricio Mora ${ }^{1}$, Alberto Vargas ${ }^{1}$, Gerardo J. Soto ${ }^{1}$, Rafael Barquero ${ }^{1}$, Wilfredo Rojas ${ }^{1}$, Waldo Taylor ${ }^{1} \&$ Magda Taylor $^{1}$}

\author{
${ }^{1}$ Red Sismológica Nacional (RSN: UCR-ICE), Apdo. 214-2060, \\ San Pedro, Costa Rica \\ ${ }^{2}$ GEOMAR Helmholtz-Zentrum für Ozeanforschung Kiel, Germany \\ *Autor para contacto: lepolt.linkimer@ucr.ac.cr
}

(Recibido: 09/07/2013 ; aceptado: 26/11/2013)

\begin{abstract}
On 5 September 2012 a magnitude Mw 7.6 earthquake struck the Nicoya Peninsula in Costa Rica. Based on two methods and using 1D and 3D velocity models, we present three earthquake location solutions, which nearly converge at the epicenter. The size, location, and the focal mechanism reported by different seismological institutions show that this earthquake was generated at the Cocos-Caribbean plate interface. An intensity map is also presented based on the intensities of 190 localities in Costa Rica for the main shock and 77 localities for the largest aftershock. The highest intensity for the main shock was VII (Modified Mercalli) at the epicentral area and in the Grecia, Sarchi, and Naranjo region. The earthquake distribution during the first month after the earthquake defines an aftershock area of about $100 \times 40 \mathrm{~km}^{2}$.

Keywords: Samara Earthquake, Nicoya peninsula, subduction, earthquake location, intensity map.

RESUMEN: El 5 de setiembre del 2012 se produjo un terremoto (Mw 7,6) en la región de la península de Nicoya. A partir de dos métodos que usan modelos de velocidades en 1D y 3D presentamos tres soluciones para el inicio de la ruptura del terremoto, los cuales convergen aproximadamente en su localización epicentral. El tamaño del evento, su localización y los parámetros de la fuente reportados por diferentes agencias sismológicas indican que el terremoto fue generado en el área interplacas, debido a la subducción de la placa del Coco bajo la placa Caribe. Se presenta un mapa de isosistas a partir de la intensidad recopilada en 190 localidades de Costa Rica para el evento principal y 77 localidades para la réplica de mayor magnitud. Las intensidades máximas para el evento principal fueron de VII (Mercalli Modificada: MM) en el área epicentral y en la zona de Grecia, Sarchí y Naranjo. El patrón de la sismicidad durante el primer mes post terremoto define un área de réplicas de $100 \times 40 \mathrm{~km}^{2}$.

Palabras clave: Terremoto de Sámara, península de Nicoya, subducción, localización de sismos, mapa de isosistas
\end{abstract}




\section{INTRODUCCIÓN}

El miércoles 5 de setiembre del 2012 se produjo un sismo $(\mathrm{Mw} 7,6)$ en la región de la península de Nicoya, el cual fue sentido en toda América Central. Después del de Limón en abril de 1991 (Mw 7,7), el terremoto del 5 de setiembre podría ser catalogado como el segundo más fuerte sucedido en territorio nacional desde el inicio de las mediciones instrumentales, en la década de 1970, por parte de las entidades que hoy conforman la Red Sismológica Nacional (RSN): la Universidad de Costa Rica (UCR) y el Instituto Costarricense de Electricidad (ICE). Este estudio contiene un análisis preliminar realizado por personal de la RSN (UCR-ICE) incluyendo una síntesis del marco tectónico e instrumental, la localización del sismo y sus efectos.

\section{CONTEXTO TECTÓNICO}

La tectónica y la sismicidad en Costa Rica son definidas en gran parte por el proceso de subducción de la placa del Coco bajo la placa Caribe. En las zonas de subducción los sismos interplacas se generan en una superficie buzante, relativamente estrecha ( 100-200 km de ancho) y somera $(<50 \mathrm{~km}$ de profundidad) conocida como "la zona sismogénica". La terminología se refiere al segmento del límite interplacas donde ocurre un deslizamiento inestable. En las últimas dos décadas, numerosos estudios geológicos y geofísicos han sido llevados a cabo en el margen pacífico de Costa Rica, en el marco de varios proyectos internacionales con participación nacional y con gran interés en delimitar la zona sismogénica y la estructura del margen (p.ej. Sallarès et al., 2001, DeShon et al., 2006, Worzewski et al., 2010, entre muchos otros). Así, se trata de una de las zonas de subducción mejor estudiadas del mundo.

Bajo la península de Nicoya se subduce un piso oceánico con una batimetría relativamente suave, que se cree promueve una zona de contacto más continua entre placas y es capaz de generar terremotos mayores a los esperados en el Pacífico Central (Protti et al., 1994). Este es parte de uno de los tres dominios morfológicos propuestos para la placa del Coco frente a la costa del Pacífico de Costa Rica (von Huene et al., 2000). Estudios tomográficos y geodésicos en el área de la península de Nicoya (p.ej. DeShon et al., 2006) revelan además que la sismicidad que ocurre entre grandes terremotos no define la zona sismogénica en su totalidad. Por ejemplo, la zona de ruptura del terremoto de 1950 (Ms 7,7) parece haberse extendido a menores y mayores profundidades (Avants et al., 2001).

\section{PRINCIPALES EVENTOS HISTÓRICOS}

En la región de Guanacaste han ocurrido al menos 15 terremotos desde el siglo XIX. De ellos los más importantes relacionados con el proceso de subducción fueron los de 1833, 1916, 1950, 1978 y 1990. El 5 de octubre de 1950 ocurrió el denominado Terremoto de Nicoya, con una magnitud $\mathrm{Mw}$ estimada entre 7,3 y 7,7 e intensidades máximas de hasta VIII (Mercalli Modificada, MM). Este terremoto provocó levantamientos de $\sim 1 \mathrm{~m}$ en la costa de la península. A pesar de su magnitud, el evento no cobró vidas humanas pero sí hubo daños en la infraestructura, principalmente en Nicoya y Puntarenas (Miyamura, 1980). Otro sismo significativo ocurrió el 25 de marzo de 1990 en la entrada al golfo de Nicoya. Afortunadamente no hubo muertes, aunque el evento causó grandes daños en estructuras civiles, deslizamientos y licuefacción (Barquero \& Boschini, 1991).

\section{DATOS DEL TERREMOTO DE SÁMARA}

Fecha: 5 de setiembre del 2012, hora local 8:42 a.m.

Localización: $15 \mathrm{~km}$ al sur-suroeste de Sámara, península de Nicoya.

Coordenadas: $9,752^{\circ} \mathrm{N}$ y $85,572^{\circ} \mathrm{W}$.

Profundidad: $9,4 \mathrm{~km}$.

Magnitud: Mw 7,6.

Mecanismo focal: Inverso puro, con planos (rumbo, buzamiento y ángulo de Rake) de: $299^{\circ}$, $18^{\circ}$ y $86^{\circ}$ y $122^{\circ}, 72^{\circ}$ y $91^{\circ}$ (de acuerdo con el USGS WPhase Moment Solution del USGS, Servicio Geológico de los Estados Unidos).

Origen: Subducción de la placa del Coco. 


\section{LOCALIZACIÓN DEL EVENTO PRINCIPAL}

La localización a posteriori fue realizada a partir de la compilación de los registros de estaciones que operaban y transmitían en tiempo real al centro de registro de la RSN en la UCR, estaciones de registro local operadas en los proyectos de generación eléctrica del ICE, y estaciones compartidas por centros sismológicos de otros países de América Central. Se incorporaron los registros de estaciones acelerográficas ubicadas en la península de Nicoya, facilitados por el Laboratorio de Ingeniería Sísmica (LIS-INII) de la UCR y varias estaciones de la red sísmica del Observatorio Vulcanológico y Sismológico de Costa Rica (OVSICORI-UNA). En Arroyo et al. (2012) se presentaron soluciones del epicentro sin utilizar las estaciones de la red del OVSICORI, que fueron incorporadas en este estudio para aumentar el número de observaciones y mejorar la cobertura (Figura 1). Se utilizaron dos técnicas para la relocalización:

1. La tradicional basada en el programa HYP, usando el modelo de velocidades 1D que emplea actualmente la RSN. Este procedimiento, a su vez, fue aplicado de dos maneras: en la primera se permitió al algoritmo identificar por sí mismo los arribos de la fase tipo Pn; en la segunda, la identificación de esta fase se llevó a cabo manualmente. Para ello se estimó una distancia de entre 100 y $110 \mathrm{~km}$ a partir de la cual se esperaría la aparición de ese tipo de fase en la configuración geométrica de la zona de subducción.

2. El programa de relocalización probabilística no lineal NonLinLoc de Lomax et al. (2000), alimentado con el modelo de velocidades 3D para Costa Rica calculado por Husen et al. (2003). El empleo de un modelo 3D tiene la indudable ventaja de que representa mejor la compleja heterogeneidad de una zona de subducción, en contraposición con una representación con capas paralelas. Sin embargo, el área epicentral del terremoto se ubica fuera del área de mejor resolución del modelo, que fue derivado solamente con estaciones en tierra. Hacia la fosa, las velocidades se mantienen cercanas al modelo 1D mínimo de velocidades de onda P para Costa Rica de Quintero
\& Kissling (2001), que fue usado como referencia en la tomografía (Husen et al., 2003). Esto podría aumentar la incertidumbre de esta relocalización.

Las tres soluciones obtenidas se presentan en el Cuadro 1 y en la Figura 1. Los epicentros de las tres localizaciones son bastante cercanos ( $\sim \mathrm{km}$ de diferencia) y el hipocentro oscila entre los 9,4 y 18,9 km. Estas diferencias son producidas principalmente por aspectos propios de las técnicas utilizadas, diferencias en los modelos de velocidad, problemas de cobertura espacial y factores inherentes a la complejidad de la ruptura ocurrida durante el sismo, además sus valores se ubican dentro del rango de incertidumbres de localización para este evento. En vista de las dificultades para determinar la distancia del primer arribo de ondas Pn con mayor exactitud y debido a la resolución limitada del modelo 3D de Husen et al. (2003) en el área epicentral, se considera la solución 1D con Pn automáticas como la oficial para la RSN (Cuadro 1, Figura 1). Más aún, la profundidad de dicha solución se acerca más a la posición del techo de la losa en esta zona según estudios anteriores (p.ej. Sallarès et al. 2001), sobre todo considerando las incertidumbres.

\section{SISMICIDAD POST-TERREMOTO}

Durante el período de un mes después del terremoto (del 5 de setiembre al 5 de octubre), la RSN localizó 920 sismos. De estos eventos se reportaron como sentidos un total de 92 (Figura 2). Durante este periodo, los sismos de mayor magnitud ocurrieron los días 8 y 15 de setiembre, con magnitudes $\mathrm{Mw}$ de 5,4 y 5,2, respectivamente.

El patrón de la sismicidad durante el primer mes, posterior al terremoto, muestra un área de gran concentración de sismos, al noreste de la isoterma de $100^{\circ} \mathrm{C}$ en el área interplacas del modelo térmico de Harris et al. (2010), temperatura que en teoría podría definir la posición del límite superior de la zona sismogénica. Esta zona de gran concetración de sismos es mostrada en la Figura 2 y puede ser interpretada como el área de réplicas originada en la zona interplacas en la cual ocurrió deslizamiento durante el terremoto. Los sismos 


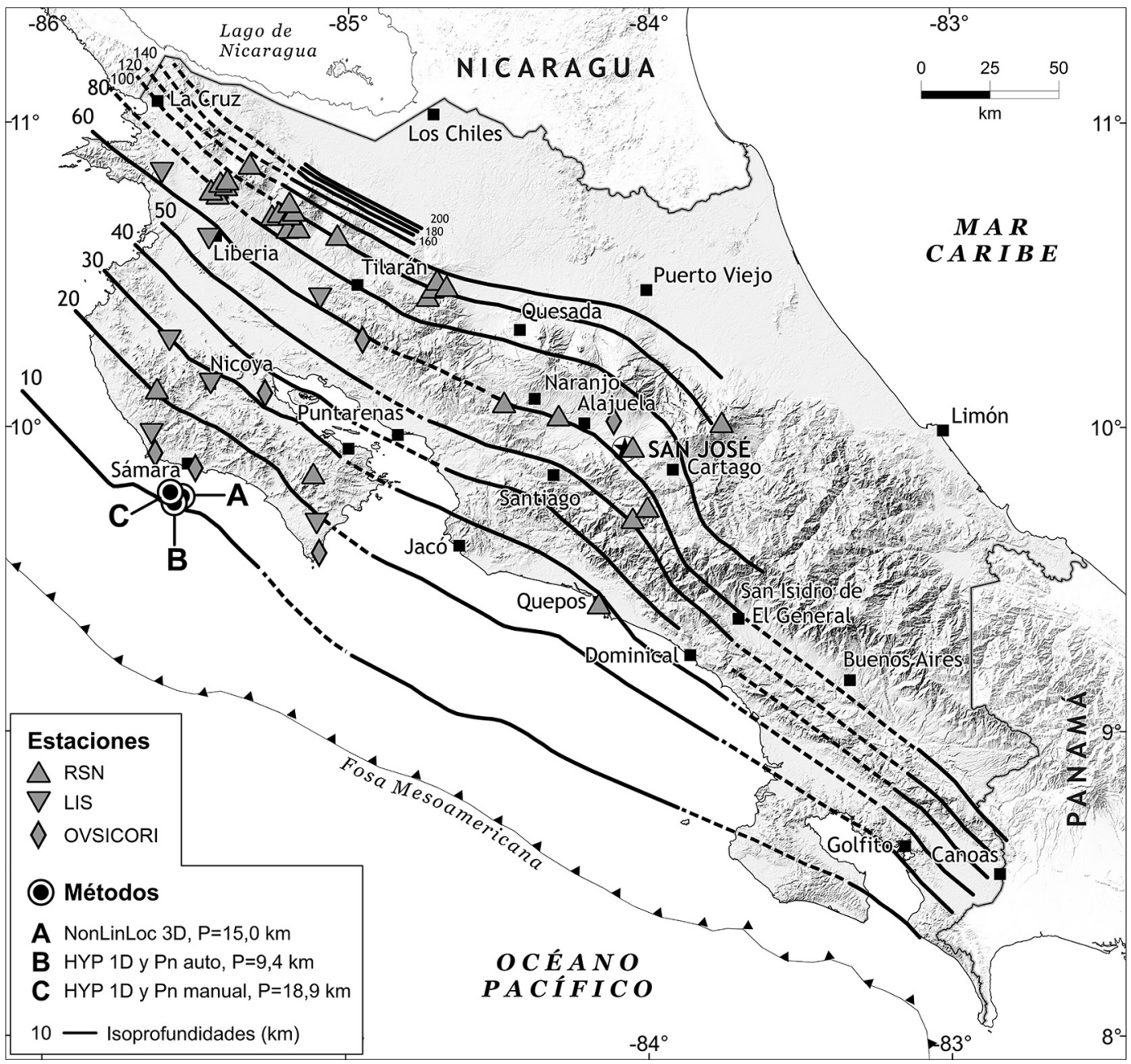

Fig. 1: Soluciones posibles del inicio de la ruptura del evento principal usando tres métodos. A Localización con un modelo 3D usando el programa NonLinLoc, B Localización con un modelo 1D usando los arribos de la fase Pn picados automáticamente y C Localización con un modelo 1D usando los arribos de la fase Pn picados manualmente (ver texto). Las estaciones empleadas en la localización se indican con diferente simbología para la RSN, el LIS y el OVSICORI-UNA. Las líneas negras son los contornos del techo de la losa sismogénica (profundidad en km; I. Arroyo, com. esc., 2013).

fuera de esta área, pueden tener otro origen ya sea en fallas en la placa cabalgante o en fallas dentro de la placa del Coco.

La zona de réplicas tiene unos $100 \mathrm{~km}$ de largo y $40 \mathrm{~km}$ de ancho en su parte más ancha y es alargada en forma paralela a la península de Nicoya. Sobresalen dos lóbulos que se extienden hacia afuera de la costa hasta la isoterma de $100^{\circ} \mathrm{C}$. Un rasgo interesante es la zona de baja sismicidad entre los dos lóbulos justo al sur del inicio de la ruptura del terremoto (epicentro). Esta zona parece coincidir con una región de bajo aclopamiento entre placas antes del terremoto, según el modelo geodésico de Feng et al. (2012). Como la sismicidad entre terremotos se inicia varios kilómetros por debajo de la isoterma de $100^{\circ} \mathrm{C}$, se podría especular que la porción superior de la zona sismogénica interplacas se encuentra en un régimen condicionalmente estable.

La mayoría de los sismos sentidos durante el primer mes luego del terremoto ocurrió en la zona de la península de Nicoya. Sin embargo, 
Cuadro 1

Soluciones calculadas para el inicio de la ruptura del evento principal

\begin{tabular}{cccccccc}
\hline Técnica & Longitud Oeste & Latitud Norte & $\begin{array}{c}\text { Profundidad } \\
(\mathrm{km})\end{array}$ & Tiempo origen & $\begin{array}{c}\text { Gap } \\
\left({ }^{\circ}\right)\end{array}$ & $\begin{array}{c}\text { Rms } \\
(\mathrm{s})\end{array}$ & $\begin{array}{c}\text { Incert. }(\mathrm{km}) \\
\text { lon, lat, prof }\end{array}$ \\
\hline $\begin{array}{c}\text { NonLinLoc 3D } \\
\begin{array}{c}\text { HYP 1D y Pn } \\
\text { automática }\end{array}\end{array}$ & 85,546 & 9,773 & 15,0 & $14: 42: 04,29$ & 216 & 0,38 & $0,40,71,2$ \\
$\begin{array}{c}\text { HYP 1D y Pn } \\
\text { manual }\end{array}$ & 85,572 & 9,752 & 9,4 & $14: 42: 03,92$ & 230 & 0,28 & $4,04,03,3$ \\
\hline
\end{tabular}

la sismicidad también se incrementó en otras zonas del país, especialmente en El GuarcoDesamparados, Tapesco-Zarcero-Atenas, Monteverde-Esparza, norte de Cartago, Zona Norte, Puriscal, Pérez Zeledón y en la zona de la isla Calero, en la frontera noreste con Nicaragua (Linkimer et al., 2012). Toda esa sismicidad se considera como inducida en otros sistemas tectónicos y no corresponde propiamente con réplicas. Asimismo, se detectó un conspicuo efecto del terremoto en las cordilleras volcánicas de Costa Rica, sobre todo en la sismicidad en sistemas tectónicos conocidos en los macizos volcánicos, reflejado en el disparo casi inmediato de una sismicidad más frecuente y con magnitudes más importantes (de hasta $\mathrm{Mw}$ 3,6) en el flanco sureste del volcán Irazú, así como otros sismos en los edificios volcánicos del Turrialba, Poás, Platanar, Arenal, Tenorio y Rincón de la Vieja (Mora et al., 2012).

\section{EFECTOS E INTENSIDADES}

El terremoto fue sentido en todo Costa Rica e inclusive en localidades de Nicaragua, Panamá, El Salvador y Guatemala. La mayor parte de los daños reportados tuvieron lugar en la zona de la península de Nicoya, particularmente en las poblaciones de Nicoya, Santa Cruz, Nandayure y Sámara (Figura 3). En Puntarenas, Mata de Limón y Jacó hubo daños en algunas construcciones, que incluyen el hospital y la Municipalidad de Puntarenas. En los cantones Grecia, Naranjo y Valverde Vega se presentaron daños importantes en viviendas y algunos edificios. En la ciudad de
San José, aunque el sismo provocó alarma general en la población, los daños no fueron severos y sólo se reportaron algunos vidrios quebrados y la caída de objetos y láminas de cielorraso en edificios. La Comisión Nacional de Prevención de Riesgos y Atención de Emergencias (CNE) informó que 240 personas estuvieron en albergues temporales habilitados para las familias afectadas en las provincias de Alajuela (34 personas) y Guanacaste (206).

El Consejo de Gobierno detalló que los diferentes ministerios e instituciones oficiales valoraron en $\notin 22360$ millones los costos de los daños en infraestructura pública y viviendas, repartidos principalmente en 20 cantones del país. El informe de la CNE indica que ocurrieron daños en 15 carreteras nacionales, 38 puentes (según un análisis preliminar del Ministerio de Obras Públicas y Transportes y el Consejo Nacional de Vialidad), 33 edificios de salud (entre hospitales, clínicas y equipos básicos de atención integral) y 1990 viviendas (en su mayoría con daños parciales). El Ministerio de Educación Pública estimó daños en 56 centros educativos, la mayoría ubicados en Nicoya (10 escuelas), Nandayure (9), Hojancha (4), Santa Cruz (9), Paquera, Lepanto, Cóbano, Sarchí, Grecia y Naranjo. El Instituto Costarricense de Acueductos y Alcantarillados (AyA) informó que siete acueductos fueron afectados principalmente en las provincias de Guanacaste y Puntarenas.

El ICE reportó daños en cinco líneas de transmisión y siete subestaciones, lo que representa un $6 \%$ de las líneas y un $12 \%$ de las subestaciones. En cuanto al sistema telefónico, el ICE informó que luego del sismo se produjo una saturación de los 


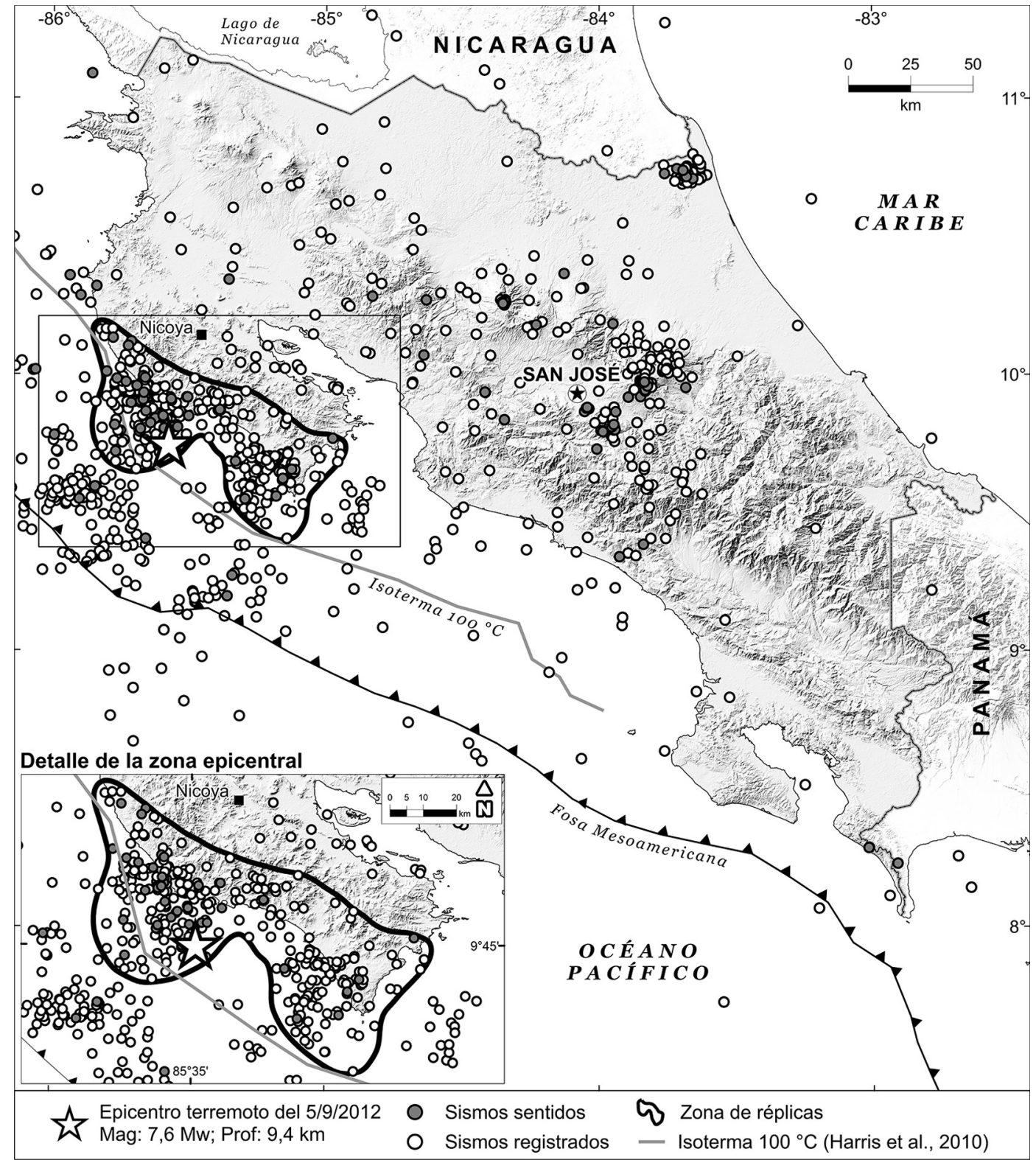

Figura 2. Mapa de localización de los sismos registrados (círculos blancos) y sentidos (círculos grises) durante el período del 5 de setiembre al 5 de octubre del 2012 en Costa Rica. La zona con la mayor concentración de réplicas ubicada al noreste de la isoterma de $100^{\circ} \mathrm{C}$ (según Harris et al., 2010) es demarcada por una línea gruesa. La estrella denota el epicentro del Terremoto de Sámara.

sistemas de telefonía fija y celular debido a que el tráfico de llamadas se cuadruplicó. Además, 104 radio bases de telefonía celular GSM y 202 de $3 \mathrm{G}$ estuvieron fuera de operación por falla en el suministro de electricidad, pero al final del día casi todos los sistemas se recuperaron. 


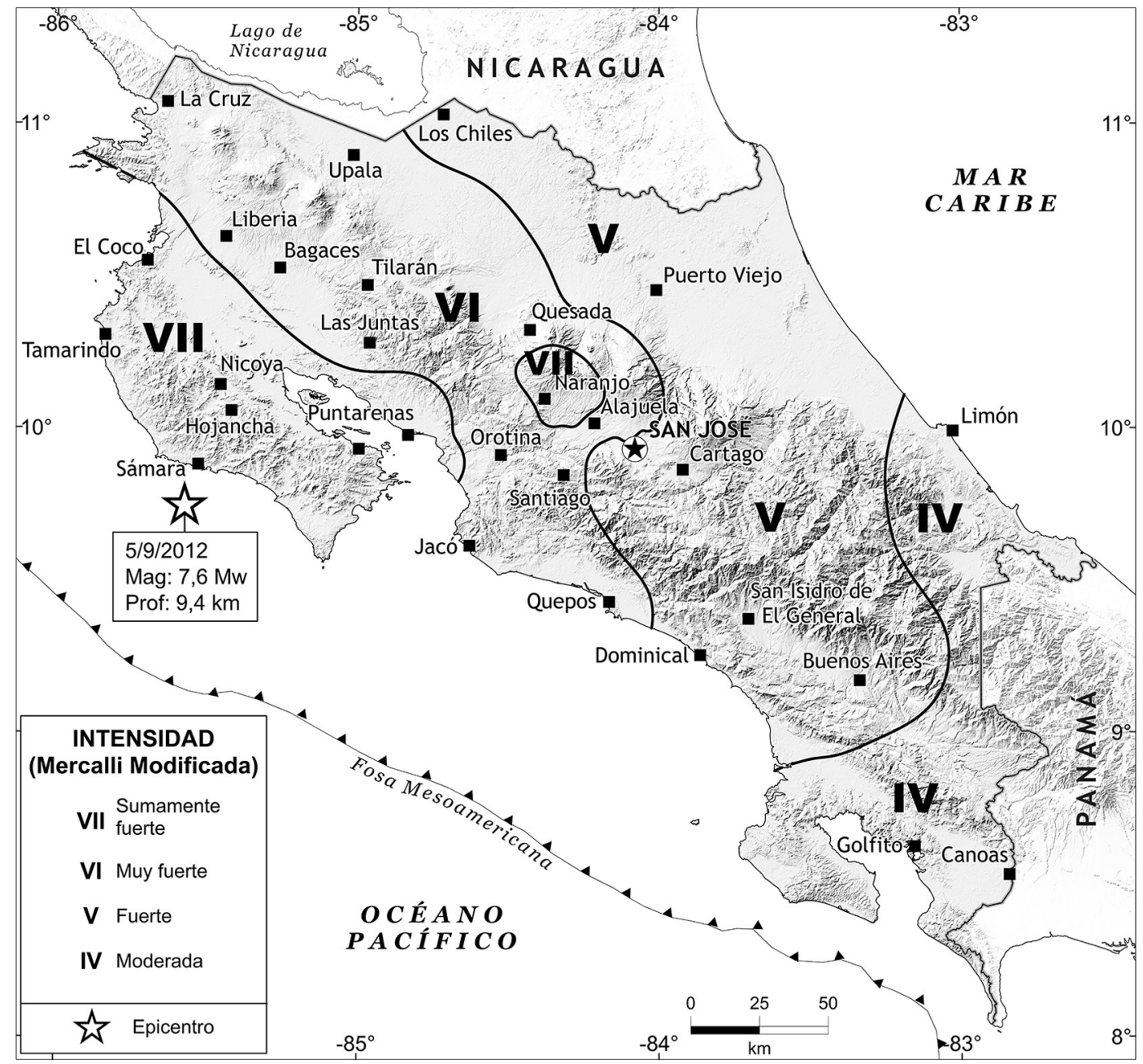

Figura 3. Mapa de intensidades en la escala Mercalli Modificada del terremoto del 5 de setiembre del 2012.

Con ayuda del reporte de los usuarios de las redes sociales que administra la RSN y de las entrevistas y efectos observados en el campo, se pudo determinar un valor de intensidad (Mercalli Modificada, IMM) en 190 localidades de Costa Rica con lo que se construyó el mapa de isosistas presentado en la figura 3. Se estimó que la intensidad máxima fue de VII (IMM, Figura 3) para la zona de la península de Nicoya y Puntarenas y además en Zarcero, Grecia y Naranjo donde se presentaron efectos de sitio (amplificación).
En Liberia, Cañas, Tilarán, Miramar y Ciudad Quesada se estimó una intensidad de VI y en gran parte del Valle Central una intensidad de V. En forma similar, se construyó el mapa de intensidades para la réplica de mayor magnitud $(\mathrm{Mw} 6,1)$ ocurrida el día 23 de octubre del 2012 a las 6:45 pm con base en la información de 77 localidades en Costa Rica (Figura 4). Para este sismo se estimó que la intensidad máxima fue de $\mathrm{V}$ en la península de Nicoya, la ciudad de Puntarenas y además en Zarcero, Grecia y Naranjo. 


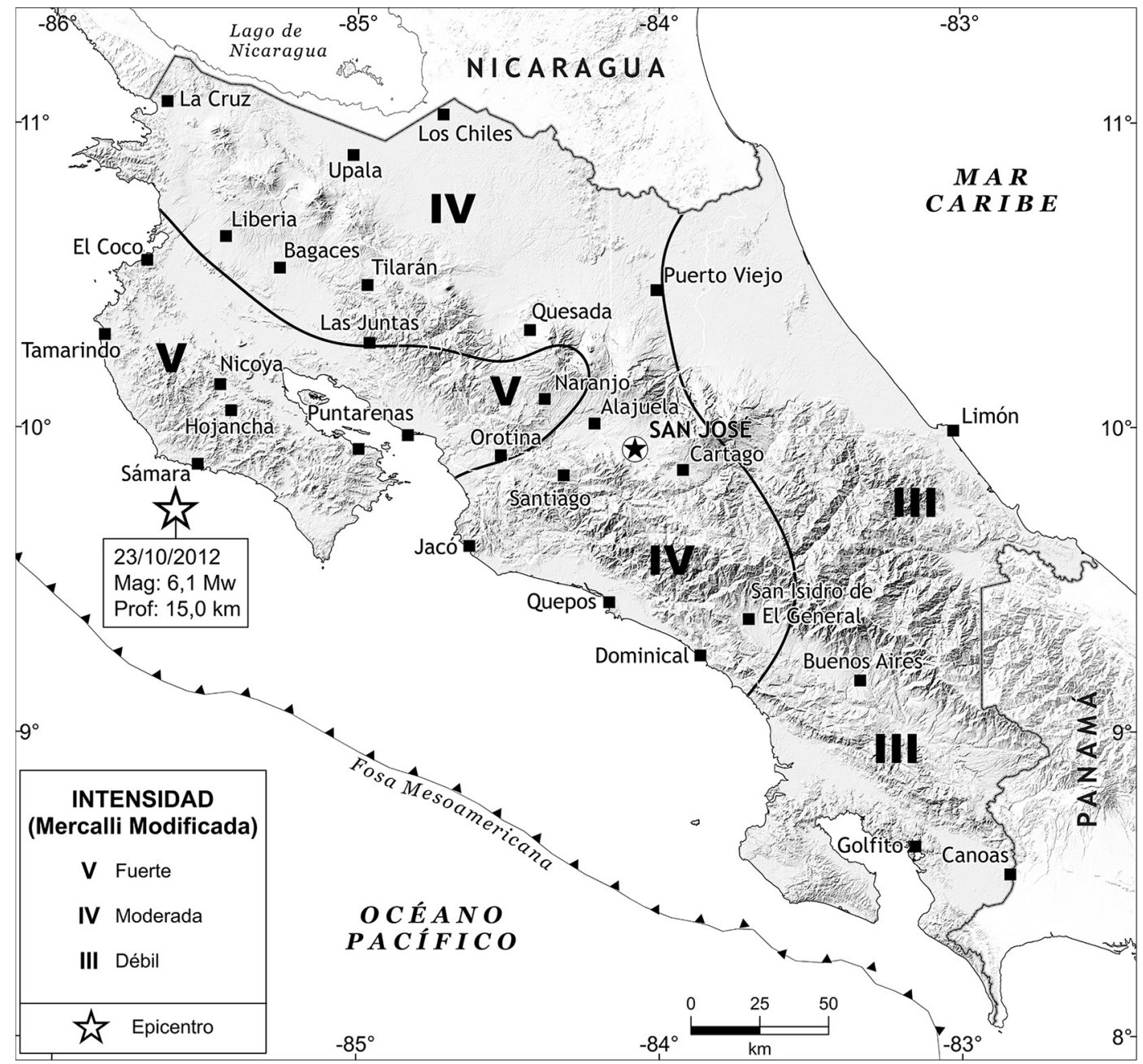

Fig. 4: Mapa de intensidades en la escala Mercalli Modificada de la réplica de mayor magnitud ocurrida el 23 de octubre del 2012.

Otros efectos del terremoto se observaron durante una inspección a la zona mesosísmica. Se determinó un levantamiento en la línea de costa de $0,45 \mathrm{~m}$ en playa Carrillo (coordenadas locales $373730 \mathrm{E}-205465 \mathrm{~N}$ ) y de $0,75 \mathrm{~m}$ en playa Pelada (coordenadas 352705 E - 215 $530 \mathrm{~N}$ ). En Ostional (coordenadas $349600 \mathrm{E}$ - $220200 \mathrm{~N}$ ) no se encontró ningún levantamiento. Además, ocurrió asentamiento diferencial y licuefacción en zonas arenosas saturadas de agua en las localidades de Carrillo, Guiones, Nosara y Ostional.

\section{AGRADECIMIENTOS}

Agradecemos al Laboratorio de Ingeniería Sísmica (LIS-INII) de la Universidad de Costa Rica y al OVSICORI de la Universidad Nacional de Costa Rica por proveer las formas de onda de algunas estaciones de la provincia de Guanacaste y al personal de la RSN de la Escuela Centroamericana de Geología de la Universidad de Costa Rica (UCR) y del Área de Amenaza y Auscultación Sísmica y Volcánica del Instituto Costarricense de Electricidad (ICE). 


\section{REFERENCIAS}

ARROYO, I.G., MORA, M. \& LINKIMER, L., 2012: Localización del evento principal.En: LINKIMER. L. \& SOTO, G.J., (eds.): El Terremoto de Sámara del 5 de setiembre del 2012.- 134 págs. Red Sismológica Nacional [Informe interno].

AVANTS, M., SCHWARTZ, S.Y., NEWMAN, A.V., DESHON, H.R., PROTTI, J.M., \& GUENDEL, F., 2001: Large underthrusting earthquakes beneath the Nicoya Peninsula, Costa Rica.- EOS, Transactions, American Geophysical Union AGU, 82: F1266.

BARQUERO, R. \& BOSCHINI, I. (eds), 1991: La crisis sísmica del Golfo de Nicoya y eventos sísmicos relacionados, Costa Rica, 1990.- 163 págs. Red Sismológica Nacional [Informe int.].

DESHON, H.R., SCHWARTZ, S.Y., NEWMAN, A.V., GONZÁLEZ, V., PROTTI, M., DORMAN, L.M., DIXON, T.H., SAMPSON, D.E. \& FLUEH, E.R., 2006: Seismogenic zone structure beneath the Nicoya Peninsula, Costa Rica, from threedimensional local earthquake P- and S-wave tomography.- Geophys. J. Int. 164(1): 109-124.

FENG, L., NEWMAN, A.V., PROTTI, M., GONZÁLEZ, V., JIANG, Y. \& DIXON, T.H., 2012: Active deformation near the Nicoya Peninsula, northwestern Costa Rica, between 1996 and 2010: Interseismic megathrust coupling.- J. Geophys. Res. 117(B6): B06407.

HARRIS, R.N., SPINELLI, G., RANERO, C.R., GREVEMEYER, I., VILLINGER, H. \& BARCKHAUSEN, U., 2010: Thermal regime of the Costa Rican convergent margin: 2. Thermal models of the shallow Middle
America subduction zone offshore Costa Rica. Geochem, Geophys, Geosyst. 11(12): Q12S29, doi:10.1029/2010gc003273.

HUSEN, S., QUINTERO, R., KISSLING, E. \& HACKER, B., 2003: Subduction-zone structure and magmatic processes beneath Costa Rica constrained by local earthquake tomography and petrological modeling.Geophys. J.,Int. 155(I):11-32.

LINKIMER, L., TAYLOR, M., ARAYA, C., BARQUERO, R. \& BOSCHINI, I., 2012: Sismicidad post-terremoto: 5 de setiembre a 5 de octubre del 2012.- En: LINKIMER. L. \& SOTO, G.J., (eds): El Terremoto de Sámara del 5 de setiembre del 2012.-134 págs. Red Sismológica Nacional [Informe int.].

LOMAX, A., VIRIEUX, J., VOLANT, P. \& BERGE-THIERRY, C., 2000: Probabilistic earthquake location in $3 \mathrm{D}$ and layered models.- En: THURBER, C.H. \& RABINOWITZ, N. (eds): Advances in seismic event location. Kluwer Academic Publishers, Dordrecht: 101-134.

MIYAMURA, S., 1980: Sismicidad de Costa Rica.- 190 págs. Ed. Univ. de Costa Rica, San José.

MORA, M.M., TAYLOR, W., \& SOTO, G.J., 2012: Sismicidad inducida y otros efectos causados en el arco magmático. - En: LINKIMER. L. \& SOTO, G.J., (eds): El Terremoto de Sámara del 5 de setiembre del 2012.- 134 págs. Red Sismológica Nacional [Informe int.].

PROTTI, M., GÜENDEL, F. \& MCNALLY, K., 1994: The geometry of the Wadati-Benioff zone under southern Central America and its tectonic significance: results from a highresolution local seismographic network.Phys. Earth Planet. Inter. 84(1-4): 271-287. 
QUINTERO, R. \& KISSLING, E., 2001: An improved P-wave velocity reference model for Costa Rica.- Geofis. Int. 40: 3-19.

SALLARÈS, V., DAÑOBEITIA, J.J. \& FLUEH, E.R., 2001: Lithospheric structure of the Costa Rican Isthmus: Effects of subduction zone magmatism on an oceanic plateau.- J. Geophys. Res. 106(B1): 621643.
VON HUENE, R., RANERO, C.R., WEINREBE, W. \& HINZ, K., 2000: Quaternary convergent margin tectonics of Costa Rica, segmentation of the Cocos Plate, and Central American volcanism.- Tectonics, 19(2): 314-334.

WORZEWSKI, T., JEGEN, M., KOPP, H., BRASSE, H. \& TAYLOR, W., 2010: Magnetotelluric image of the fluid cycle in the Costa Rican subduction zone.- Nature Geosci. 4(2): 108-111. 